\title{
Tropism of pandemic 2009 H1N1 influenza A virus
}

\section{Ririn Ramadhany ${ }^{1,2}$, Mayo Yasugi ${ }^{2 \dagger}$, Shota Nakamura, ${ }^{3,4}$, Tomo Daidoji ${ }^{1,5}$, Yohei Watanabe ${ }^{2}$, Kazuo Takahashi ${ }^{6}$, Kazuyoshi Ikuta ${ }^{2}$ and Takaaki Nakaya ${ }^{1,4,5 *}$}

1 International Research Center for Infectious Diseases, Research Institute for Microbial Diseases, Osaka University, Suita, Osaka, Japan

2 Department of Virology, Research Institute for Microbial Diseases, Osaka University, Suita, Osaka, Japan

${ }^{3}$ Department of Genome Informatics, Research Institute for Microbial Diseases, Osaka University, Suita, Osaka, Japan

${ }^{4}$ Department of Infection Metagenomics, Research Institute for Microbial Diseases, Osaka University, Suita, Osaka, Japan

${ }^{5}$ Department of Infectious Diseases, Kyoto Prefectural University of Medicine, Kamigyo-ku, Kyoto, Japan

${ }^{6}$ Osaka Prefectural Institute of Public Health, Higashinari-ku, Osaka, Japan

\section{Edited by:}

Akio Adachi, The University of

Tokushima Graduate School, Japan

Reviewed by:

Dale L. Barnard, Utah State

University, USA

Kiyoko Iwatsuki-Horimoto, University

of Tokyo, Japan

\section{*Correspondence:}

Takaaki Nakaya, International

Research Center for Infectious

Diseases, Research Institute for

Microbial Diseases, Osaka University,

3-1 Yamadaoka, Suita, Osaka

565-0871, Japan; Department of

Infectious Diseases, Kyoto Prefectural

University of Medicine, 465

Kawaramachi-hirokoji, Kamigyo-ku,

Kyoto 602-8566, Japan.

e-mail: tnakaya@biken.osaka-u.ac.jp

tnakaya@koto.kpu-m.ac.jp

${ }^{t}$ Present address:

Mayo Yasugi, Graduate School of Life and Environmental Sciences, Osaka

Prefecture University, Izumisano,

Osaka, Japan.
Substitutions at the receptor-binding site of the pandemic H1N1 2009 influenza A virus $(\mathrm{H} 1 \mathrm{~N} 1 \mathrm{pdm})$ hemagglutinin $(\mathrm{HA})$ gene may be critical in determining whether a virus binds to human or avian receptors. Previous reports suggest that HA Gly 222 and/or $\operatorname{Arg}^{223}$ allow viruses to bind preferentially to the $\alpha 2,3$-linked sialic acid found in avian species. We also demonstrated that serial passaging of influenza $A$ virus in embryonated chicken eggs increased viral growth 32- to 64-fold, coincident with the increased prevalence of Gly ${ }^{222}$ or $\mathrm{Arg}^{223}$ in HA protein (Yasugi et al., 2012). In this study, we showed that the minor genotype of $\alpha 2,3$-linkage-tropic viruses in upper airways became dominant after passaging through chicken eggs. Viruses possessing HA containing N125D-Q223R, N125D-D187E-O223R, K119N-D222G, and K119N-N129S-D222G, were detected in both clinical specimens and egg-passaged samples. These results might suggest that egg-adapted viruses, likely represented by $\alpha 2,3$-linkage-tropic virus, were also present in human upper airways as a minor population and transmitted in humans during the outbreak of H1N1pdm.

Keywords: influenza virus, H1N1pdm, next generation sequencer, genetic diversity, D222G

\section{INTRODUCTION}

Eight segments of a novel swine-derived H1N1 2009 influenza A virus (H1N1pdm) genome are derived from classical North American and Eurasian swine lineages, an avian lineage, and from human $\mathrm{H} 3 \mathrm{~N} 2$. The hemagglutinin (HA) gene is thought to be derived from classical North American swine (Cohen, 2009). The receptor-binding specificity of HA is responsible for the host range restriction of influenza A virus. Human and classical H1N1 swine influenza viruses bind preferentially to the glycosylated sialic acid (SA) linkage $\alpha 2,6 \mathrm{Gal}$, whereas most avian viruses have higher binding affinity for SA $\alpha 2,3 \mathrm{Gal}$ (Nicholls et al., 2008). Receptor specificity is determined by the nature of the amino acids that form the receptor-binding pocket of HA. Gln at position 223 and Gly at position 225 found in avian virus isolates determine the specificity for SA $22,3 \mathrm{Gal}$ (Stevens et al., 2004), whereas Leu and Ser at these positions confer SA $\alpha 2,6 \mathrm{Gal}$ specificity in $\mathrm{H} 2$ and H3 viruses (Gamblin et al., 2004). For H1 viruses, Asp (found in human viruses) or Glu (found in avian viruses) at position 187 determine preferential binding to $\alpha 2,6$ or $\alpha 2,3$ linkages, respectively (Gambaryan et al., 1999). The amino acids at positions 135, 191, and 222 also affect receptor-binding affinity and specificity
(Rogers et al., 1983; Vines et al., 1998; Matrosovich et al., 2000; Chen et al., 2010).

Since the first appearance of H1N1pdm viruses, substitutions including D222G in HA protein and the $\mathrm{K} 340 \mathrm{~N}$ substitution in RNA polymerase basic 2 (PB2) have appeared sporadically (WHO Global Alert and Responses, 2009). We investigated the nucleotide diversity of the 2009 H1N1pdm HA gene during the first (May 2009) and second (Dec 2010) waves of the epidemic in Japan, with particular focus on the receptor-binding site (RBS). Sequencing analyses using conventional and next-generation high-throughput approaches showed that there were quasispecies in the HA genome, including D222G, in humans (Yasugi et al., 2012). In this study, we focused on the shift of genotype(s) of $\alpha 2,3$-linkage-tropic viruses in upper airways to become dominant after passaging through chicken eggs.

\section{MATERIALS AND METHODS CLINICAL SAMPLES}

Nasal swab samples used in this study were obtained from mild H1N1pdm cases in Osaka, Japan at different time points. Three 
and five samples (\#1 to \#3: first wave in May 2009 and \#4 to \#8: second wave in December 2010, respectively) were randomly selected (Yasugi et al., 2012). The analyzed samples were unlinked and anonymous at Osaka Prefectural Institute of Public Health. This study was approved by the ethics review committees of Osaka Prefectural Institute of Public Health and the Research Institute for Microbial Diseases (RIMD; Yasugi et al., 2012), Osaka University. Both ethics committees specifically waived the need for consent.

\section{RNA EXTRACTION}

Each nasal swab collected in Hanks solution was centrifuged at $20,000 \times g$ for $10 \mathrm{~min}$. The supernatants were suspended in TRIzol LS reagent (Invitrogen) for $60 \mathrm{~min}$. Total RNA was extracted with a PureLink RNA Mini Kit (Invitrogen) according to the manufacturer's instructions. Contaminating DNA was eliminated with DNAase I (Invitrogen).

\section{DIRECT SEQUENCING}

Total RNA was subjected to one-step RT-PCR (SuperScript III/Platinum Taq One-step RT-PCR Kit; Invitrogen). Primer sets used to amplify each viral genome segment are shown in Table A1 in Appendix. PCR products were purified using a MonoFas DNA purification kit (GL Sciences) and nucleotide sequences were obtained using a BigDye Terminator v3.1 Cycle Sequencing Kit (Applied Biosystems).

\section{VIRUS ISOLATION AND SERIAL PASSAGE IN EMBRYONATED EGGS AND MADIN-DARBY CANINE KIDNEY CELLS}

Clinical specimens were injected into 9-day-old embryonated chicken eggs for virus isolation (Daidoji et al., 2008). After incubation at $37^{\circ} \mathrm{C}$ for $72 \mathrm{~h}$, the allantoic fluids were collected and filtered (Passage 0: P0). The samples were then diluted 1- to 100fold in phosphate buffered saline (PBS) and passaged either five (\#3) or six times (\#1, \#2, and \#4 to \#8) in embryonated chicken eggs. Identical specimens derived from \#1 to \#2 were also inoculated into MDCK cells for virus isolation (Ueda et al., 2010) and the amplified viruses were serially passaged two or three times in MDCK cells.

\section{HEMAGGLUTINATION TITRATION}

Viral samples were serially diluted with PBS and added at a concentration of $0.5 \%$ to chicken red blood cells. After incubation at room temperature for $30 \mathrm{~min}$, hemagglutination was observed.

\section{HIGH-THROUGHPUT PYROSEQUENCING ANALYSIS}

Total RNA was subjected to one-step RT-PCR using the primer set for HA-RBS (Yasugi et al., 2012). A total of $6 \mu \mathrm{g}$ amplified DNA was subjected to high-throughput pyrosequencing analysis (Roche/454 GS-FLX Titanium system). A $70 \times 75$ PicoTiterPlate device (gasket for 16 regions) was divided into 16 regions and one region was used for the mixed samples. Data analysis was performed on each sequence read using computational tools as described previously (Nakamura et al., 2008, 2009).

\section{RESULTS}

The genotype of the egg-passaged viruses (P5 in \#3 or P6 in $\# 1$ and \#2) derived from the first wave was analyzed. Amplicons representing each segment of the P5 and P6 viral genomes were PCR amplified with gene-specific primers (Table A1 in Appendix) and the nucleotide sequence of each PCR product was directly determined by traditional sequencing. No amino acid substitutions were detected in the PB2, RNA polymerase basic 1 (PB1 and PB1-F2), RNA polymerase acidic (PA), neuraminidase (NA), matrix proteins (M1 and M2), and non-structural proteins (NS1 and NS2) compared with A/Osaka/01/2009 (Table 1; Yasugi et al., 2012). Passaged 1- and 3-derived viruses exhibited one amino acid mutation in the nucleoprotein (NP), but not at the same site (Table 1), suggesting that the mutation was not directly correlated to egg adaptation. In contrast, several mutations (E66K, K119N, N125D, D222G, Q223R, and N370K) in the HA were detected and K119N, D222G, and Q223R were abundant substitutions in multiple specimen-derived viruses (Table $\mathbf{1}$ ). The sequencing data also showed that D222G and/or Q223R substitutions became dominant in the population of egg-passaged viruses, suggesting that the shift in HA genotype contributed to the adaptation of the passaged viruses to avian cells.

We performed high-throughput sequencing analysis by GSFLX Titanium pyrosequencing. PCR products amplifying the same region (HA-RBS) as shown previously (Yasugi et al., 2012), which covers almost all the above mutations, except E66K and N370K, were prepared from egg-passaged viruses $\mathrm{P} 1$ and $\mathrm{P} 5$ or $\mathrm{P} 6$. The substitution rate of six critical amino acids (K119N, N125D, N129S, D187E, D222G, Q223R; Yasugi et al., 2012) and A215V is summarized in Tables 2-4. Viruses passaged five or six times in eggs (P5, P6) showed higher mutation rates than the parental viruses isolated from clinical specimens. HA sequences harboring N125D, D187E, and Q223R mutations found in the \#1-P6 viruses (Table 2), K119N, N125D, N129S, D187E, D222G, and Q223R found in

Table 1 | Direct Sanger sequencing of RT-PCR products amplified from each viral genome segment in egg-passaged viruses.

\begin{tabular}{|c|c|c|c|c|c|c|}
\hline \multirow[t]{2}{*}{ Segment } & \multicolumn{2}{|c|}{ \#1 (P6) } & \multicolumn{2}{|c|}{ \#2 (P6) } & \multicolumn{2}{|c|}{ \#3 (P5) } \\
\hline & nt & aa & nt & aa & nt & aa \\
\hline PB2 & 0 & 0 & 0 & 0 & 0 & 0 \\
\hline PB1* & 0 & 0 & 0 & 0 & 0 & 0 \\
\hline PA & 0 & 0 & 0 & 0 & 0 & 0 \\
\hline \multirow[t]{3}{*}{$H A^{*}$} & G427A & E66 K & G408T & K119N & G408T & K119N \\
\hline & A424G & N125D & A716G & D222G & A716G & D222G \\
\hline & A719G & Q223R & A719G & Q223R & T1161A & N370K \\
\hline \multirow[t]{2}{*}{ NP } & T159G & D53E & 0 & 0 & G447A & D375N \\
\hline & & & & & G1123A & \\
\hline NA & 0 & 0 & 0 & 0 & 0 & 0 \\
\hline M & 0 & 0 & 0 & 0 & 0 & 0 \\
\hline NS & 0 & 0 & 0 & 0 & 0 & 0 \\
\hline
\end{tabular}

P6 generation was studied for \#1 and \#2-derived viruses and the P5 generation was studied for the \#3-derived viruses.

The results show nucleotide (nt) and amino acid (aa) substitutions compared with A/Osaka/01/2009.

${ }^{*}$ R634H found within PB1 and 1142V found within HA were commonly detected in all egg-adapted viruses as well as the viruses in the clinical specimen (\#2). 
Table 2 | Frequency of multiple mutations in nine amino acids of the H1N1pdm HA in egg-passaged (P1 and P6), and MDCK-passaged samples derived from \#1.

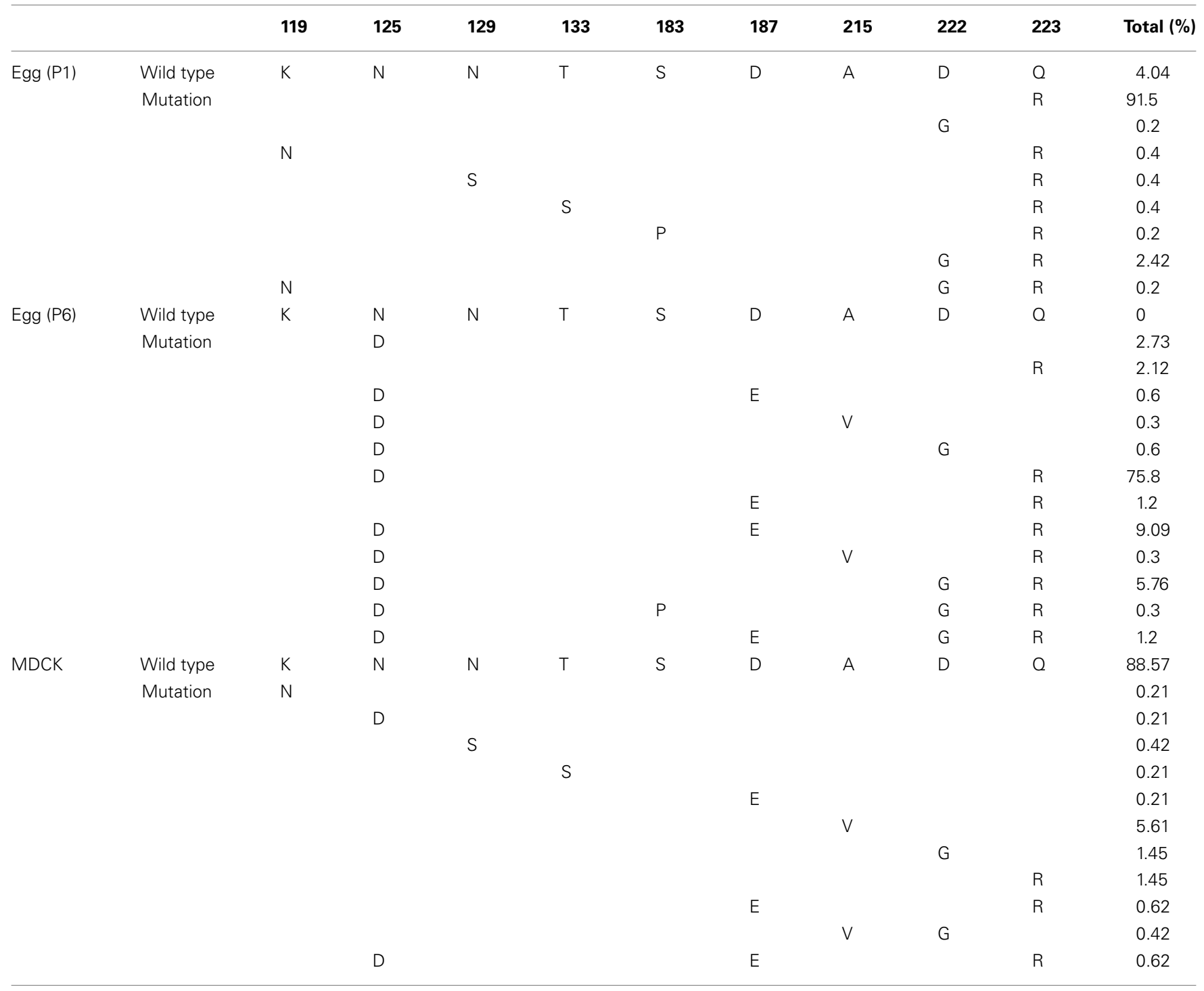

Critical substitutions such as K119N, N125D, N129S, D187E, A215V, D222G, Q223R, and two additional substitutions T133S and S183P, as controls, are shown.

the \#2-P6 viruses (Table 3), and K119N, N125D, N129S, A215V, and $\mathrm{D} 222 \mathrm{G}$ mutations found in the \#3-P5 viruses predominated (Table 4). The N125D-Q223R (76\%) genotype derived from \#1 and K119N-N129S-D222G (45.5\%), and N125D-D187E-Q223R (21.8\%) genotypes derived from \#2 and K119N-A215V-D222G (39\%), N125D-D222G (17\%), and N125D-N129S-D222G (11\%) genotypes derived from \#3 were also detected as major populations (Tables 2-4). The shift in HA genotype through passaging in eggs is assessed in Figure 1. Of particular interest, sequences containing double and/or triple amino acid mutations, which were abundantly detected in egg-passaged viruses, were also found as a minor population in its nasal specimen. For example, HA harboring N125D-D187E-Q223R (Table 2; Figure 1), K119N-N129SD222G and N125D-Q223R (Table 3; Figure 1), or K119N-D222G (Table 4) was detected in \#1, \#2, and \#3 clinical samples (Yasugi et al., 2012), respectively. These results suggest that egg-adapted viruses, likely represented by $\alpha 2,3$-linkage-tropic virus, were also present in human upper airways as a minor population and transmitted in humans during the outbreak of H1N1pdm. Because $2,6 \mathrm{Gal}$ expression is abundant and 2,3Gal expression is minor in epithelial cells of the upper respiratory tracts (Shinya et al., 2006), it might make sense that $\alpha 2,3$-linkage-tropic viruses exist as a minor population in human upper airways.

In contrast to the first wave, both the G222 and R223 variants had almost disappeared (0.01-0.07 and 0.47-0.63\%, respectively) in five nasal swabs (\#4 to \#8) obtained from individuals with a mild case of H1N1pdm in December 2010 (second wave; Yasugi et al., 2012). We then inoculated the five specimens (\#4 to \#8) into embryonated chicken eggs and serially passaged them six times in eggs. Hemagglutination was faintly detected in samples in the 
Table 3 | Frequency of multiple mutations in nine amino acids of the H1N1pdm HA in egg-passaged (P1 and P6), and MDCK-passaged samples derived from \#2.

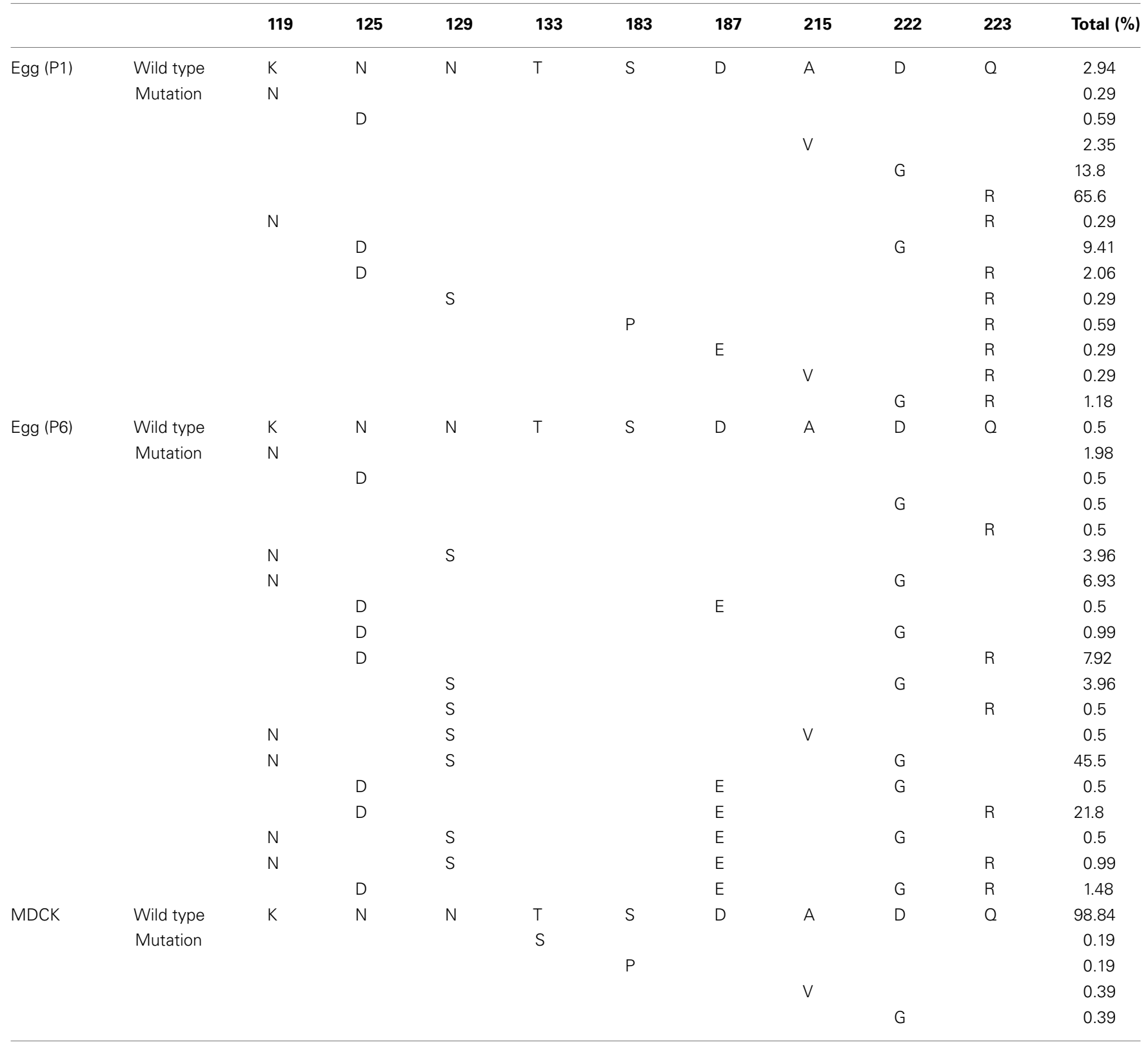

Critical substitutions such as K119N, N125D, N129S, D187E, A215V, D222G, Q223R, and two additional substitutions T133S and S183P, as controls, are shown.

first passage (P0) but was detected in the third or fourth passage and showed $2^{5}-2^{7}$ hemagglutination titers in the fifth or sixth passage (P5 or P6) in the four specimens except \#8 (Table 5). PCR products amplifying the same region as \#1 to \#3 (first wave; Yasugi et al., 2012) were prepared and were examined using conventional direct PCR sequencing. The G222 (\#4, \#6) and R223 (\#5 and \#7) variants were detected even in P1 passages in the four specimens (Table 6), although both variants had very minor populations in nasal specimens. In addition, no other variants, except the above two amino acids, were detected (data not shown); thus, further high-throughput sequencing analysis was not performed in \#4 to $\# 8$.

\section{DISCUSSION}

Previous work demonstrated that D222G and/or Q223R mutation led to the binding of $\alpha 2,3-S A-$ resialylated chicken red blood cells (Chen et al., 2010). D222G and/or Q223R mutations might also be crucial for the tropism and/or pathogenesis of H1N1pdm. The function(s) of the other substitutions shown in Tables 2-4 are largely unknown; however, significant similarities in the HA genotype between the viruses resident in upper airways (Yasugi et al., 2012) and egg-adapted viruses (Tables 2-4) suggest that the minor genotype of $\alpha 2,3$ linkage-tropic viruses in upper airways became dominant after passaging through chicken eggs. Viruses derived from \#1 and 
Table 4 | Frequency of multiple mutations in nine amino acids of the H1N1pdm HA in egg-passaged (P1 and P5) samples derived from \#3.

\begin{tabular}{|c|c|c|c|c|c|c|c|c|c|c|c|}
\hline & & 119 & 125 & 129 & 133 & 183 & 187 & 215 & 222 & 223 & Total (\%) \\
\hline & Mutation & $\mathrm{N}$ & & & & & & & & & 0.33 \\
\hline & & & & & & & & & $\mathrm{G}$ & & 57.3 \\
\hline & & & & & & & & & & $\mathrm{R}$ & 0.67 \\
\hline & & $N$ & & & & $P$ & & & & & 0.33 \\
\hline & & & & & & $P$ & & & $\mathrm{G}$ & & 1.33 \\
\hline \multirow[t]{14}{*}{ Egg (P5) } & Wild type & $K$ & $\mathrm{~N}$ & $N$ & $\mathrm{~T}$ & $\mathrm{~S}$ & $D$ & $A$ & $\mathrm{D}$ & $\mathrm{O}$ & 1.03 \\
\hline & Mutation & $N$ & & & & & & & & & 2.07 \\
\hline & & & $\mathrm{D}$ & & & & & & & & 2.07 \\
\hline & & & & & & & & & $\mathrm{G}$ & & 4.66 \\
\hline & & & & & & & & & & $\mathrm{R}$ & 0.52 \\
\hline & & & $\mathrm{D}$ & $S$ & & & & & & & 1.55 \\
\hline & & & $\mathrm{D}$ & & & & & & $\mathrm{G}$ & & 17.1 \\
\hline & & & & $S$ & & & & & $\mathrm{G}$ & & 0.52 \\
\hline & & & & & & & & V & $\mathrm{G}$ & & 8.29 \\
\hline & & $\mathrm{N}$ & & & & & & V & $\mathrm{G}$ & & 38.9 \\
\hline & & & $D$ & $S$ & & & & V & & & 1.55 \\
\hline & & & $D$ & $S$ & & & & & $\mathrm{G}$ & & 10.9 \\
\hline & & & $D$ & & & & & V & $\mathrm{G}$ & & 0.52 \\
\hline & & & $\mathrm{D}$ & $S$ & & & & V & $\mathrm{G}$ & & 2.07 \\
\hline
\end{tabular}

Critical substitutions such as K119N, N125D, N129S, D187E, A215V, D222G, Q223R, and two additional substitutions T133S and S183P, as controls, are shown.

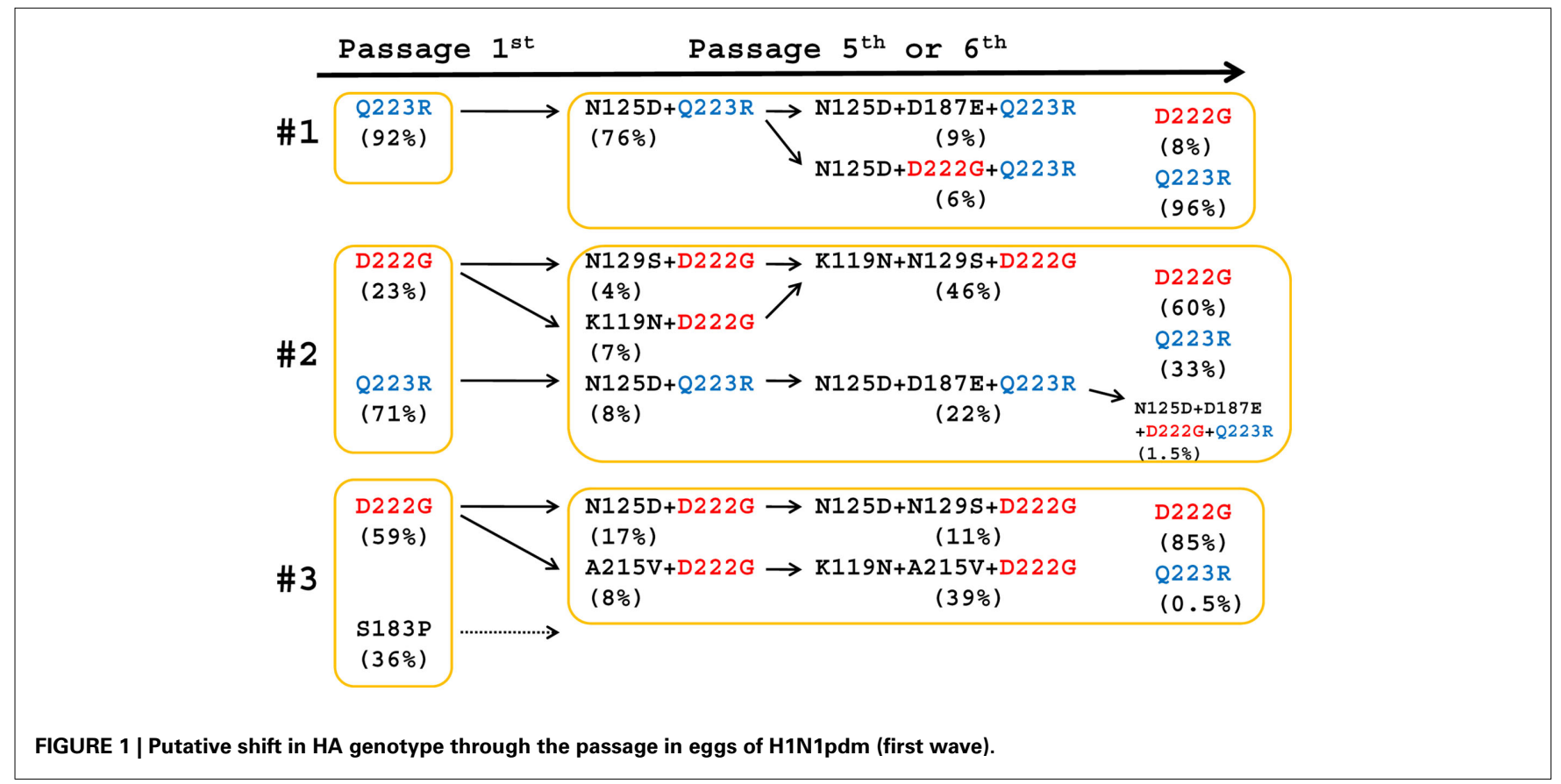

possessing HA containing N125D-Q223R and N125D-D187EQ223R, viruses derived from \#2 containing N125D-Q223R and
K119N-N129S-D222G, and viruses derived from \#3 containing K119N-D222G and N125D-S207N-D222G, were detected in both 
Table 5 | HA titer of virus second wave H1N1pdm samples passaged in eggs.

\begin{tabular}{lccccccc}
\hline Sample & \multicolumn{7}{c}{ HA titer (2 Log 10) } \\
\cline { 2 - 8 } & P0 & P1 & P2 & P3 & P4 & P5 & P6 \\
\hline \#4 & 1 & 1 & 1 & 5 & 4 & 5 & 4 \\
$\# 5$ & 1 & 6 & 7 & 7 & 6 & 7 & 7 \\
$\# 6$ & 1 & 1 & 7 & 5 & 5 & 5 & 3 \\
$\# 7$ & 1 & 7 & 7 & 5 & 6 & 6 & 5 \\
$\# 8$ & 1 & 1 & 1 & 0 & 0 & 0 & 0
\end{tabular}

Table 6 | D222G and Q223R mutations on second wave H1N1pdm samples.

\begin{tabular}{lllllll}
\hline Sample & \multicolumn{6}{c}{ Passage } \\
\cline { 2 - 7 } & P0 & P1 & P3 & P4 & P5 & P6 \\
\hline$\# 4$ & No & D222G & D222G & No & D222G & No \\
$\# 5$ & No & Q223R & Q223R & Q223R & Q223R & Q223R \\
$\# 6$ & ND & D222G & D222G & D222G & D222G & D222G \\
$\# 7$ & No & Q223R & Q223R & Q223R & Q223R & Q223R
\end{tabular}

No, no mutation on 222 and 223 position.

$N D$, not done.

${ }^{*}$ Double peak = double peak as in GGT (wild type) and GAT (D to G) on position 222.

clinical specimens and egg-passaged samples (Figure 1; Yasugi et al., 2012). These results suggest a direct linkage of $\alpha 2,3$-tropic viruses between the clinical samples and egg-adapted samples in each case.

However, such a direct linkage could not be observed in the second wave of H1N1pdm, because the G222 and R223 variants were almost undetected (0.01-0.07 and $0.47-0.63 \%$, respectively) in five nasal swabs (\#4 to \#8; Yasugi et al., 2012). Thus, D222G or Q223R mutation may occur in inoculated eggs. Further investigations of clonal recombinant virus using reverse genetics are required to address this question. We also failed to rescue eggadapted virus from \#8 (Table 5). In addition, neither D222G nor Q223R genotype was detected in the sixth passage of \#4 (Table 6), although egg-adapted viruses well grew (Table 5). These results might suggest that factor(s) other than mutation(s) in HA-RBS (Lu et al., 2005) are involved in egg adaptation.

We showed that MDCK-passaged virus isolates contain a similar ratio of $\alpha 2,3$-tropic and $\alpha 2,6$-tropic viruses compared to

\section{REFERENCES}

Audsley, J. M., and Tannock, G. A. (2008). Cell-based influenza vaccines: progress to date. Drugs 68, 1483-1491.

Chen, Z., Wang, W., Zhou, H., Suguitan, A. L. Jr., Shambaugh, C., Kim, L., Zhao, J., Kemble, G., and Jin, H. (2010). Generation of live attenuated novel influenza virus A/California/7/09 (H1N1) vaccines with high yield in embryonated chicken eggs. J. Virol. 84, 44-51.

Cohen, J. (2009). Swine flu outbreak. Out of Mexico? Scientists ponder swine flu's origins. Science 324, 700-702.

the original H1N1pdm (first wave) viruses identified in human specimens (Tables 2 and 3; Yasugi et al., 2012). Takemae et al. (2010) demonstrated that embryonated chicken egg-isolated classical H1 swine influenza viruses harbored substitutions including $\mathrm{D} 187 \mathrm{~V} / \mathrm{N}$ and $\mathrm{D} 222 \mathrm{G}$ in the HA, whereas MDCK isolates retained HA genes identical to those of the viruses present in the swine nasal swab samples. Passaging in MDCK cells may therefore be a better approach to establish genetic diversity and specific HA genotypes in vivo in human and swine influenza viruses. While egg adaptation is currently required to prepare vaccine candidates, alternative approaches involving the use of accredited anchorage-dependent and -independent preparations of the African Green monkey kidney (Vero), MDCK, and other cell lines have been pursued by several manufacturers in recent years (Audsley and Tannock, 2008). Our results, in this study, suggest the advantage of the cell-based influenza vaccine approach, which is able to maintain the genetic stability of clinical strains.

This study, together with our previous report (Yasugi et al., 2012), suggest that $\alpha 2,3-S A-s p e c i f i c$ viruses, including G222 and $\mathrm{R} 223$, existed in humans as a minor population in the early phase (first wave) of the pandemic, and that D222 and Q223 became more dominant through human-to-human transmission (second wave) during the epidemic. Newly emergent influenza A viruses may have been dual specific but not exclusively $\alpha 2,6-\mathrm{SA}$-specific during the early phase of the pandemic and adapted during multiple cycles of human-to-human transmission (Yasugi et al., 2012). Further investigation is required to determine the proportion of $\alpha 2,3$-tropic and $\alpha 2,6$-tropic viruses found in tissues and organs infected with other human-, avian-, and swine-derived influenza viruses. Deep sequencing approaches will provide a more comprehensive analysis of genetic diversity in egg- and MDCK-passaged viruses and original virus isolates in vivo and will help us to understand quasispecies of influenza viruses more precisely.

\section{ACKNOWLEDGMENTS}

We thank Dr. Yoshinobu Okuno, The Research Foundation for Microbial Diseases of Osaka University for helpful discussions, and Ms. Atsuyo Yoshioka, RIMD, Osaka University, for technical assistance. This work was supported in part by a Grant-in-Aid for Scientific Research from the Ministry of Education, Science, Sports, Culture, and Technology (MEXT) to Takaaki Nakaya; a Grant-in-Aid for Young Scientists from the Japan Society for the Promotion of Science (JSPS) to Tomo Daidoji, Mayo Yasugi, and Ririn Ramadhany is a research fellow of Hashiya Scholarship Foundation.

Daidoji, T., Koma, T., Du, A., Yang, C. S., Ueda, M., Ikuta, K., and Nakaya, T. (2008). H5N1 avian influenza virus induces apoptotic cell death in mammalian airway epithelial cells. J. Virol. 82, 11294-11307.

Gambaryan, A. S., Robertson, J. S., and Matrosovich, M. N. (1999). Effects of egg-adaptation on the receptor-binding properties of human influenza A and B viruses. Virology 258, 232-239.

Gamblin, S. J., Haire, L. F., Russell, R. J., Stevens, D. J., Xiao, B., Ha, Y., Vasisht, N., Steinhauer, D. A., Daniels, R. S., Elliot, A., Wiley, D. C., and Skehel, J. J. (2004). The structure and receptor binding properties 
of the 1918 influenza hemagglutinin. Science 303, 1838-1842.

Lu, B., Zhou, H., Ye, D., Kemble, G., and Jin, H. (2005). Improvement of influenza A/Fujian/411/02 (H3N2) virus growth in embryonated chicken eggs by balancing the hemagglutinin and neuraminidase activities, using reverse genetics. $J$. Virol. 79, 6763-6771.

Matrosovich, M., Tuzikov, A., Bovin, N., Gambaryan, A., Klimov, A., Castrucci, M. R., Donatelli, I., and Kawaoka, Y. (2000). Early alterations of the receptor-binding properties of $\mathrm{H} 1, \mathrm{H} 2$, and $\mathrm{H} 3$ avian influenza virus hemagglutinins after their introduction into mammals. J. Virol. 74, 8502-8512.

Nakamura, S., Maeda, N., Miron, I. M., Yoh, M., Izutsu, K., Kataoka, C., Honda, T., Yasunaga, T., Nakaya, T., Kawai, J., Hayashizaki, Y., Horii, T., and Iida, T. (2008). Metagenomic diagnosis of bacterial infections. Emerging Infect. Dis. 14, 1784-1786.

Nakamura, S., Yang, C. S., Sakon, N., Ueda, M., Tougan, T., Yamashita, A., Goto, N., Takahashi, K., Yasunaga, T., Ikuta, K., Mizutani, T., Okamoto, Y., Tagami, M., Morita, R., Maeda, N., Kawai, J., Hayashizaki, Y., Nagai, Y., Horii, T., Iida, T., and Nakaya, T.
(2009). Direct metagenomic detection of viral pathogens in nasal and fecal specimens using an unbiased high-throughput sequencing approach. PLoS ONE 4, e4219. doi:10.1371/journal.pone.0004219

Nicholls, J. M., Chan, R. W., Russell, R. J., Air, G. M., and Peiris, J. S. (2008). Evolving complexities of influenza virus and its receptors. Trends Microbiol. 16, 149-157.

Rogers, G. N., Paulson, J. C., Daniels, R. S., Skehel, J. J., Wilson, I. A., and Wiley, D. C. (1983). Single amino acid substitutions in influenza haemagglutinin change receptor binding specificity. Nature 304, 76-78.

Shinya, K., Ebina, M., Yamada, S., Ono, M., Kasai, N., and Kawaoka, Y. (2006). Avian flu: influenza virus receptors in the human airway. Nature 440, 435-436.

Stevens, J., Corper, A. L., Basler, C. F., Taubenberger, J. K., Palese, P., and Wilson, I. A. (2004). Structure of the uncleaved human $\mathrm{H} 1$ hemagglutinin from the extinct 1918 influenza virus. Science 303, 1866-1870.

Takemae, N., Ruttanapumma, R., Parchariyanon, S., Yoneyama, S., Hayashi, T., Hiramatsu, H., Sriwilaijaroen, N., Uchida, Y., Kondo,
S., Yagi, H., Kato, K., Suzuki, Y., and Saito, T. (2010). Alterations in receptor-binding properties of swine influenza viruses of the $\mathrm{Hl}$ subtype after isolation in embryonated chicken eggs. J. Gen. Virol. 91, 938-948.

Ueda, M., Daidoji, T., Du, A., Yang, C. S., Ibrahim, M. S., Ikuta, K., and Nakaya, T. (2010). Highly pathogenic $\mathrm{H} 5 \mathrm{~N} 1$ avian influenza virus induces extracellular $\mathrm{Ca} 2+$ influx, leading to apoptosis in avian cells. J. Virol. 84, 3068-3078.

Vines, A., Wells, K., Matrosovich, M., Castrucci, M. R., Ito, T., and Kawaoka, Y. (1998). The role of influenza A virus hemagglutinin residues 226 and 228 in receptor specificity and host range restriction. J. Virol. 72, 7626-7631.

WHO Global Alert and Responses. (2009). Preliminary Review of D222G Amino Acid Substitution in the Haemagglutinin of Pandemic Influenza A (H1N1) 2009 Viruses.

Yasugi, M., Nakamura, S., Daidoji, T., Kawashita, N., Ramadhany, R., Yang, C. S., Yasunaga, T., Iida, T., Horii, T., Ikuta, K., Takahashi, K., and Nakaya, T. (2012). Frequency of D222G and Q223R hemagglutinin mutants of pandemic (H1N1) 2009 influenza virus in Japan between 2009 and 2010. PLoS ONE 7, e30946. doi:10.1371/journal.pone.0030946

Conflict of Interest Statement: The authors declare that the research was conducted in the absence of any commercial or financial relationships that could be construed as a potential conflict of interest.

Received: 23 January 2012; accepted: 19 March 2012; published online: 04 April 2012.

Citation: Ramadhany $R$, Yasugi $M$, Nakamura S, Daidoji T, Watanabe Y, Takahashi $K$, Ikuta $K$ and Nakaya $T$ (2012) Tropism of pandemic 2009 H1N1 influenza A virus. Front. Microbio. 3:128. doi: 10.3389/fmicb.2012.00128

This article was submitted to Frontiers in Virology, a specialty of Frontiers in Microbiology.

Copyright (ङ) 2012 Ramadhany, Yasugi, Nakamura, Daidoji, Watanabe, Takahashi, Ikuta and Nakaya. This is an open-access article distributed under the terms of the Creative Commons Attribution Non Commercial License, which permits non-commercial use, distribution, and reproduction in other forums, provided the original authors and source are credited. 


\section{APPENDIX}

Table A1 | Primer pairs used to amplify segments of the H1N1pdm genome.

\begin{tabular}{|c|c|c|}
\hline \multirow[t]{2}{*}{ PB2-5 } & $\mathrm{F}$ & TGTAAAACGACGGCCAGTATGGAGAGAATAAAAGAACTGAGAG \\
\hline & $\mathrm{R}$ & GCTTGTCCTTTTGAAAGTGAACCCA \\
\hline \multirow[t]{2}{*}{ PB2-3' } & $\mathrm{F}$ & TGCAAAAGTGCTTTTCCAGAACTGG \\
\hline & $\mathrm{R}$ & CAGGAAACAGCTATGACCCTAATTGATGGCCATCCGAATTC \\
\hline \multirow[t]{2}{*}{ PB1-5' } & $\mathrm{F}$ & TGTAAAACGACGGCCAGTATGGATGTCAATCCGACTCTAC \\
\hline & $\mathrm{R}$ & ATTTTCATTCCACTTAGTGTTGTCC \\
\hline PB1-middle & $\mathrm{F}$ & CAAAGATGCAGAGAGAGGCAAG \\
\hline PB1-3' & $\mathrm{R}$ & CAGGAAACAGCTATGACCTTATTTTTTGCCGTCTGAGTTCTTCAATGGTGG \\
\hline \multirow[t]{2}{*}{ PA-5 } & $\mathrm{F}$ & TGTAAAACGACGGCCAGTATGGAAGACTTTGTGCGACAATG \\
\hline & $\mathrm{R}$ & GTCCTCAAGAATGGTTCAATTTTGG \\
\hline \multirow[t]{2}{*}{ PA-middle } & $\mathrm{F}$ & ATGGATTCGAGCCGAACGGCTGCATTG \\
\hline & $\mathrm{R}$ & TATGTACACTCCCTTCATTATGTATTCAG \\
\hline \multirow[t]{2}{*}{ HA-3' } & $\mathrm{F}$ & GTGCTATAAACACCAGCCTYCCA \\
\hline & $\mathrm{R}$ & CAGGAAACAGCTATGACCTTAAATACATATTCTACACTGTAGAG \\
\hline \multirow[t]{2}{*}{ NP } & $\mathrm{F}$ & TGTAAAACGACGGCCAGTATGGCGTCTCAAGGCACCAAACG \\
\hline & $\mathrm{R}$ & CAGGAAACAGCTATGACCTCAACTGTCATACTCСTCTG \\
\hline \multirow[t]{2}{*}{$N A-5^{\prime}$} & $\mathrm{F}$ & TGTAAAACGACGGCCAGTATGAATCCAAACCAAAAGATAATAACCATTG \\
\hline & $\mathrm{R}$ & AGAATCAGGATAACAGGAGC \\
\hline \multirow[t]{2}{*}{ NA-3' } & $\mathrm{F}$ & GAATGTGCATGTGTAAATGG \\
\hline & $\mathrm{R}$ & CAGGAAACAGCTATGACCTTACTTGTCAATGGTAAATGGCAACTCAG \\
\hline \multirow[t]{2}{*}{ M } & $\mathrm{F}$ & TGTAAAACGACGGCCAGTAGCAAAAGCAGGTAGAT \\
\hline & $\mathrm{R}$ & CAGGAAACAGCTATGACCAGTAGAAACAAGGTAGTTT \\
\hline
\end{tabular}

